\title{
THERMODYNAMIC EQUIVALENCE BETWEEN THE LENNARD-JONES AND HARD-CORE ATTRACTIVE YUKAWA SYSTEMS
}

\author{
Y. KADIRI ${ }^{a}$, R. ALBAKI ${ }^{a}$ and J. L. BRETONNET ${ }^{b}$ \\ ${ }^{a}$ Collège Ahunstic, BP 352, Montréal H2M 1 Y8, Québec, Canada \\ ${ }^{b}$ Laboratoire de Physique des Milieux Denses, Université de Metz, 1, bd F. D. Arago, \\ 57078 Metz Cedex 3, France
}

Received 4 April 2007; Accepted 4 July 2007

Online 12 July 2007

Thermodynamic properties of the Lennard-Jones (LJ) fluid are investigated by studying a system of particles interacting with a potential of hard-core plus attractive Yukawa tail (HCY). Due to the similarity of the LJ and the HCY potentials in their overall form, it is worthwhile seeking to approximate the LJ potential in much the same way that the hard-sphere reference potential has been used. The study consists in describing the thermodynamics of the LJ fluid in terms of the equivalent HCY system, whose properties are known accurately, by means of mapping the thermodynamic quantities for the HCY potential parameters. The method is feasible owing to a convenient analytical expression for the Helmholtz free energy in the mean-spherical approximation expanded in powers of the inverse temperature. Two different procedures are used to determine the parameters of the HCY potential as a function of the thermodynamic states: one is based on the simultaneous fits of pressure and internal energy of the LJ system, and the other uses the concept of collision frequency. The reasonable homogeneity of the results in both procedures of mapping makes the HCY potential a very good reference system whose theoretical expressions can be used confidently to predict the thermodynamic properties of systems with more realistic potentials.

PACS numbers: $61.20 . \mathrm{Gy}, 65.90 .+\mathrm{i}$,

UDC 536.632, 538.953

Keywords: Lennard-Jones fluid, thermodynamic properties, hard-core plus attractive Yukawa potential

\section{Introduction}

In the theory of simple fluids, one of the major achievements has been the recognition of the quite distinct roles played by the repulsive and attractive parts of the interatomic potential in determining the microscopic properties of simple fluids 
$[1,2]$. In recent years, much attention has also been paid in developing analytically solvable models suitable to represent the thermodynamic and structural properties of real fluids. The hard-sphere (HS) model is the natural reference system for describing the general features of liquids, namely the local atomic order according to the excluded volume effects and the freezing process of the fluid into an ordered solid structure. In contrast, the HS model is not able to predict the condensation of a gas into a liquid, which is only made possible by the existence of dispersion forces giving rise to an attractive long-range part in the potential. Van der Waals provided the first analytical excursion, from idealized model, in attempting to account for the finite size of the ionic core and for an inverse sixth-power attractive potential at long range.

Another system that was found very useful to stabilize the local fluid structure is the hard-core potential with an attractive Yukawa tail (HCY). It is the advantage to tune to hypothetical and real fluids of widely different properties [3] by varying its hard-sphere diameter $\sigma$ and its inverse screening length $\lambda$. For example, it is known that the charged hard-core fluid modelling systems, like colloidal suspensions and protein solutions, can be investigated with an inverse screening length $\lambda \sim 8$, while the Lennard-Jones potential parametrized for rare gases can be simulated with an inverse screening length $\lambda \sim 2$.

An additional reason that makes the HCY system appealing is that semianalytical and analytical solutions are available. The original solution given by Waisman [4] within the mean-spherical approximation (MSA) involved a set of six algebraic equations with six parameters, but progressively valuable simplifications have been found $[5-7]$ giving a reduced number of unknown parameters and simpler analytical expressions for the thermodynamic properties and the pair-correlation function. For the purpose of this work, the expressions for these properties will be used in expanded forms in powers of the inverse temperature, as derived by Henderson et al. $[8,9]$.

Such an equation of state for the HCY system, which is founded on the perturbation theory and expressed in terms of the relevant features of the potential, is a very handy tool for investigating the thermodynamics of liquids governed by an effective hard-sphere interaction plus an attractive tail. Specifically, this analytical equation of state is useful to explore how the properties of the HCY system differ from those of other systems. Then, the investigation consists in determining the HCY system equivalent to the original system, i. e. the values of the hard-sphere diameter $\sigma$ and the inverse screening length $\lambda$ for all thermodynamic states, in such a way that the thermodynamic properties of the HCY and original systems are identical. The idea of equivalence between two systems has already been used by several authors with diverse procedures of mapping [11-14]. In particular, ways of handling this concept have been considered in mapping the repulsive part of the LJ potential into an effective hard-sphere interaction [15] or in mapping the full LJ potential into the square-well potential [16]. The approximation of the LJ potential by a two-Yukawa potential has also been done along the liquid-vapour coexistence curve [17], but nothing seems to have been done to map the LJ system into the HCY one over a wide domain of the phase diagram. The method is feasible 
because the opportunity can be taken of both the similarity of the repulsive parts and the resemblance of the long-range parts of the HCY and LJ systems. It is well known that the structure factor of fluids can be fairly well predicted with the HCY model - if not with the HS model, while deficiencies that affect the pair-correlation function in the region of the first peak cannot be ignored in comparing the HCY and LJ systems. Alternatively, the HCY system could be used indirectly to approximate any available interatomic potential for a real fluid without reference to the LJ potential.

The aim of this paper is to show that the $\mathrm{HCY}$ system is a reliable reference system for describing the features of real fluids, at least so good as the squarewell system [16] in dealing with realistic LJ-type systems. The paper is organized as follows. In Section 2, the hard core potential with attractive Yukawa tail is briefly presented with the essential analytical expressions for the thermodynamic properties. Section 3 is devoted to the procedures of mapping for which it is clearly shown that the LJ potential is commonly used with constant parameters while the HCY potential is used with state-dependent hard-sphere diameter $\sigma$ and inverse screening length $\lambda$. Section 4 presents a set of values of the parameters $\sigma$ and $\lambda$ as functions of temperature and density, as well as results for the thermodynamic properties obtained by the mapping procedures. Finally, some concluding remarks hinged on the previous results are stated.

\section{Hard-core attractive Yukawa fluid as reference system}

Consider the three-dimensional fluid with the pair potential consisting of the hard-sphere core and the attractive Yukawa tail

$$
u_{\mathrm{HCY}}(r)=\left\{\begin{array}{cc}
\infty & r<\sigma \\
-(\varepsilon \sigma / r) \exp [-\lambda(r / \sigma-1)] & r>\sigma
\end{array}\right.
$$

where $\sigma$ is the hard-sphere diameter, $\varepsilon$ is the depth of the potential at the hard-core distance and $\lambda$ is a positive parameter that measures the spatial decay rate. The HCY has been studied in detail by many authors $[5,6,8]$ after the solution of the mean-spherical approximation was obtained by Waisman [4]. According to Ginoza [7], the solution reduces to determining a fundamental parameter $\Gamma$ as the root of the quartic equation

$$
\Gamma(1+\lambda \Gamma)(1+\psi \Gamma)^{2}+\beta \varepsilon w=0
$$

where $\varepsilon$ and $\lambda$ are the two parameters of the HCY potential, $w$ and $\psi$ are two supplementary parameters depending explicitly on $\lambda$ and packing fraction $\eta\left(=\pi \rho \sigma^{3} / 6\right), \rho$ being as usual the number density, and $\beta=\left(k_{B} T\right)^{-1}$.

Expanding $\Gamma$ in powers of the inverse temperature, Henderson et al. [9] obtained a polynomial expression for the free energy and, subsequently, Duh and Mier-YTeran [21] remarked that the polynomial exhibits a binomial-like trend allowing to

FIZIKA A (Zagreb) 16 (2007) 2, 47-62 
KADIRI ET AL.: THERMODYNAMIC EQUIVALENCE BETWEEN THE LENNARD-JONES AND ...

write the free energy $A$ in the following form

$$
\frac{\beta A}{N}=\frac{\beta A_{\mathrm{HS}}}{N}-\frac{\alpha_{0}}{\phi_{0}}(\beta \varepsilon)-\frac{\lambda^{3}}{6 \eta}\left[F(x)-F(y)-(x-y) \frac{\mathrm{d} F(y)}{\mathrm{d} y}\right],
$$

where the first term of the right-hand side corresponds to the hard-sphere fluid properties known analytically. $A_{\mathrm{HS}}$ used in this work comes from the Kolafa-Boublik [18] equation of state (EOS), which gives results slightly better than the most popular Carnahan-Starling [19] EOS when compared to the Monte Carlo experiments of Erpenbeck and Wood [20]. The hard-sphere free energy $\beta A_{\mathrm{HS}} / N$ is given by

$$
\frac{\beta A_{\mathrm{HS}}}{N}=\ln \Lambda^{3} \rho-1+\frac{5}{3} \ln (1-\eta)+\frac{\left(34 \eta-33 \eta^{2}+4 \eta^{3}\right)}{6(1-\eta)^{2}},
$$

where $\Lambda$ is the thermal de Broglie wavelength. The terms in the brackets of Eq. (3) involve the function $F(X)$ of arbitrary variable $X$ that reads

$$
F(X)=-\frac{1}{4} \ln (1-2 X)-2 \ln (1-X)-\frac{3}{2} X-\frac{1}{1-X}+1,
$$

and

$$
\begin{aligned}
x & =\frac{(1+\lambda \psi) w}{\lambda^{2}}(\beta \varepsilon), \\
y & =\left(\frac{w \psi}{\lambda}\right)(\beta \varepsilon) .
\end{aligned}
$$

The coefficients $w, \psi, \alpha_{0}, \phi_{0}$ can be expressed in terms of the two parameters of the HCY potential $\eta$ and $\lambda$ through the intermediate parameters

$$
\begin{aligned}
w & =\frac{6 \eta}{\phi_{0}^{2}} \\
\psi & =\lambda^{2}(1-\eta)^{2} \frac{[1-\exp (-\lambda)]}{L(\lambda) \exp (-\lambda)+S(\lambda)}-12 \eta(1-\eta) \frac{[1-\lambda / 2-(1+\lambda / 2) \exp (-\lambda)]}{L(\lambda) \exp (-\lambda)+S(\lambda)} \\
\phi_{0} & =\frac{L(\lambda) \exp (-\lambda)+S(\lambda)}{\lambda^{3}(1-\eta)^{2}} \\
\alpha_{0} & =\frac{L(\lambda)}{\lambda^{2}(1-\eta)^{2}}
\end{aligned}
$$

with

$$
\begin{aligned}
L(\lambda) & =12 \eta[1+2 \eta+(1+\eta / 2) \lambda] \\
S(\lambda) & =(1-\eta)^{2} \lambda^{3}+6 \eta(1-\eta) \lambda^{2}+18 \eta^{2} \lambda-12 \eta(1+2 \eta) .
\end{aligned}
$$


Analytical expressions can be obtained for the excess internal energy and the pressure by differentiation, in a straightforward manner, from the standard relationships

$$
\begin{aligned}
\frac{\beta U^{e x}}{N} & =\beta \frac{\partial}{\partial \beta}\left(\frac{\beta A}{N}\right)-\frac{3}{2} \\
\frac{\beta p}{\rho} & =\eta \frac{\partial}{\partial \eta}\left(\frac{\beta A}{N}\right) .
\end{aligned}
$$

It is also worth mentioning that series expansion for the pair-correlation function $g(r)$ has been derived by Henderson et al. [10]. These analytical expressions have been tested using the molecular dynamic simulation data and exact MSA results of [22] for some state points. Table 1 displays results for the excess internal energy, virial pressure and pair-correlation function at contact of the HCY system as a function of density $\rho^{*}\left(=\rho \sigma^{3}\right)$ for $T^{*}=1 /(\beta \varepsilon)=1$ and $\lambda=1.8$. The comparison between the analytical theory (AT) and molecular dynamics (MD) is particularly good for high densities. The main advantage this EOS is to determine the excess properties of the $\mathrm{HCY}$ system and to connect rigorously the parameters of the $\mathrm{HCY}$ system to the molecular properties.

Table 1. Excess internal energy, virial pressure and pair-correlation function at contact of the $\mathrm{HCY}$ fluid as a function of density, for $\lambda=1.8$ and $T^{*}=1$. The analytical theory (AT) data are compared with those of molecular dynamics (MD) and mean spherical approximation (MSA) of Ref. [22].

\begin{tabular}{|c|c|c|c|c|c|c|c|c|c|}
\hline \multirow{2}{*}{$\rho^{*}$} & \multicolumn{3}{|c|}{$\beta E^{e x} / N$} & \multicolumn{3}{c|}{$\beta p_{M C} / \rho$} & \multicolumn{3}{c|}{$g\left(\sigma^{+}\right)$} \\
\cline { 2 - 9 } & $D M$ & $M S A$ & $A T$ & $D M$ & $M S A$ & $A T$ & $D M$ & $M S A$ & $A T$ \\
\hline 0.3 & -2.114 & & -2.052 & 0.02 & & 0.014 & 2.780 & & 2.527 \\
0.4 & -2.745 & -2.665 & -2.677 & -0.20 & -0.229 & -0.207 & 2.811 & 2.280 & 2.643 \\
0.5 & -3.384 & -3.345 & -3.337 & -0.28 & -0.337 & -0.337 & 2.952 & 2.410 & 2.846 \\
0.6 & -4.079 & -4.050 & -4.051 & -0.24 & -0.283 & -0.265 & 3.228 & 2.684 & 3.173 \\
0.7 & -4.838 & -4.813 & -4.813 & +0.19 & +0.143 & 0.160 & 3.685 & 3.098 & 3.669 \\
0.8 & -5.638 & -5.616 & -5.616 & +1.17 & +1.137 & 1.157 & 4.382 & 3.681 & 4.398 \\
\hline
\end{tabular}

\section{Procedures of mapping for the Lennard-Jones system}

Restricting the discussion to simple centro-symmetric interactions from the outset, we consider the realistic interaction potential of Lennard-Jones (12-6) given by

$$
u_{\mathrm{LJ}}(r)=4 \varepsilon_{\mathrm{LJ}}\left[\left(\frac{\sigma_{\mathrm{LJ}}}{r}\right)^{12}-\left(\frac{\sigma_{\mathrm{LJ}}}{r}\right)^{6}\right] .
$$

FIZIKA A (Zagreb) 16 (2007) 2, 47-62 
Such a functional form gives a reasonable representation of the interactions operating in real fluids, with the well depth $\varepsilon_{\mathrm{LJ}}$ and the collision diameter $\sigma_{\mathrm{LJ}}$ independent of density and temperature. Figure 1 compares the LJ potential to the HCY potential of same well depth $\left(\varepsilon=\varepsilon_{\mathrm{LJ}}\right)$ and same collision diameter $\left(\sigma=\sigma_{\mathrm{LJ}}\right)$.

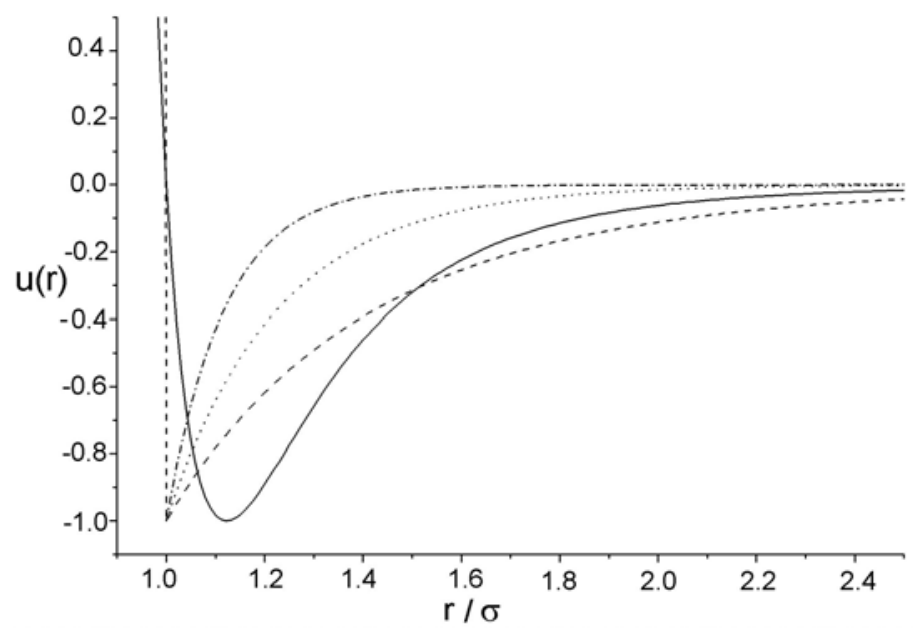

Fig. 1. HCY pair potential for different values of inverse screening length $(\lambda=1.5$ dashed line, $\lambda=3.5$ dotted line, $\lambda=7.5$ dash-dotted line), in comparison with the $L J$ system of same diameter and well depth..

Each substance has its own values of $\varepsilon_{\mathrm{LJ}}$ and $\sigma_{\mathrm{LJ}}$ so that, in a reduced form, the LJ potentials have not only the same shape for all simple fluids, but rigorously superimpose each other. This is the condition for substances to conform to the principle of corresponding states [23]. With the assumption that a fluid is made up of particles interacting with the LJ potential, the partition function and many other thermodynamic properties can be written in terms of reduced temperature $T_{\mathrm{LJ}}^{*}=k_{B} T / \varepsilon_{\mathrm{LJ}}$ and reduced density $\rho_{\mathrm{LJ}}^{*}=\rho \sigma_{\mathrm{LJ}}^{3}$ only. In particular, the virial pressure can be expressed in the implicit form [1]

$$
\frac{\beta p_{\mathrm{LJ}}}{\rho}=f_{\mathrm{LJ}}\left(T_{\mathrm{LJ}}^{*}, \rho_{\mathrm{LJ}}^{*}\right)
$$

As far as the HCY potential is concerned, the virial pressure is given by

$$
\frac{\beta p_{\mathrm{HCY}}}{\rho}=f_{\mathrm{HCY}}\left(T^{*}, \rho^{*}, \lambda\right),
$$

where $T^{*}\left(=k_{B} T / \varepsilon\right), \rho^{*}\left(=\rho \sigma^{3}\right)$ and $\lambda$ are the reduced parameters of the HCY potential, as defined in Eq. (1).

In order to determine the HCY system equivalent to the LJ system at a given $T$ and $\rho$, it is necessary to choose the values of the relevant parameters $\varepsilon, \sigma$ and $\lambda$ 
to be used in the equations for the thermodynamic properties. As there are many combinations for the values of the parameters giving the same pressure in both systems, restrictive conditions have to be imposed. The natural constraints consist in posing $\varepsilon=\varepsilon_{\mathrm{LJ}}$ and $\rho^{*}=\rho_{\mathrm{LJ}}^{*} \alpha^{3}$, where $\alpha=\sigma / \sigma_{\mathrm{LJ}}$, so that the pressure of the HCY system becomes

$$
\frac{\beta p_{\mathrm{HCY}}}{\rho}=f_{\mathrm{HCY}}\left(T_{\mathrm{LJ}}^{*}, \rho_{\mathrm{LJ}}^{*} \alpha^{3}, \lambda\right)
$$

and that the two remaining parameters to be determined are $\alpha$ and $\lambda$. These parameters are state dependent contrarily to those of the LJ potential. Hence, the procedure of mapping is reduced to the choice of the parameters $\sigma$ (or $\alpha$ ) and $\lambda$. As an example of mapping, the HCY potential could be used with a state-dependent diameter $\sigma(T, \rho)$ obtained in the same manner as the hard-sphere potential has been used by Verlet and Weis [15] to approximate the LJ potential. Then, the parameter $\lambda(T, \rho)$ could be fitted by using some reasonable fitting procedure to give a good approximation of the dispersion potential tail. Nevertheless, though the HCY potential outside the core is similar in its overall form to the LJ potential, one can expect that this method is not prudent because of the radically different functional forms of the long-ranged HCY and LJ potentials. Alternatively, two other procedures of mapping have been used in this work to determine the parameters $\sigma$ and $\lambda$ as a functions of the thermodynamic states; one uses a fine adjustment of the parameters $\sigma$ and $\lambda$ at a given $T$ and $\rho$ in fitting virial pressure and internal energy of the HCY system into those of the LJ system, and the other is based on the concept of collision frequency proposed and tested by del Rio and coworkers $[11,24,16]$.

(i) The first procedure employed to determine $\sigma$ (or $\alpha$ ) and $\lambda$ consists in fitting simultaneously the virial pressure and the excess internal energy of the HCY system into those of the LJ system. At selected thermodynamic states, the excess internal energy and pressure of the HCY system are identified, respectively, with those of the LJ system, so that $\alpha$ and $\lambda$ can be adjusted by iterations until the following equations are simultaneously satisfied

$$
\begin{aligned}
& \frac{\beta U_{\mathrm{HCY}}^{e x}\left(T_{\mathrm{LJ}}^{*}, \rho_{\mathrm{LJ}}^{*} \alpha^{3}, \lambda\right)}{N}=\frac{\beta U_{\mathrm{LJ}}^{e x}\left(T_{\mathrm{LJ}}^{*}, \rho_{\mathrm{LJ}}^{*}\right)}{N} \\
& \frac{\beta p_{\mathrm{HCY}}\left(T_{\mathrm{LJ}}^{*}, \rho_{\mathrm{LJ}}^{*} \alpha^{3}, \lambda\right)}{\rho}=\frac{\beta p_{\mathrm{LJ}}\left(T_{\mathrm{LJ}}^{*}, \rho_{\mathrm{LJ}}^{*}\right)}{\rho} .
\end{aligned}
$$

The interesting aspect of this mapping procedure is that the sets of points representative of the excess internal energy and virial pressure constancy are located on two smooth lines in the plane $(\alpha, \lambda)$ and that the uniqueness of their point of intersection provides the HCY system with the appropriate values of $\alpha$ and $\lambda$, at a given $T$ and $\rho$. It should be mentioned that the slopes of both curves are generally opposite in sign so that the point of intersection is obtained without difficulty. However, at temperatures below the critical point, the slope of the isobar line reverses as the 
density is decreased, and that is why no point of intersection can be found in the range of the metastable states where the two curves are more or less parallel.

(ii) The result of the second procedure of mapping is the following. For systems with purely repulsive potential, it is well known that the non-ideal contribution to the pressure is proportional to the rate of collisions between particles [2]. Extending their investigation to systems with realistic potentials, del Rio and Gil-Villegas [24] introduced two collision rates related to the repulsive and attractive parts of the potential, and proposed a mapping between two equivalent thermodynamic systems in such a way that, respectively, the repulsive and attractive contributions to the pressure are kept the same in both systems. This can be readily accomplished with the pressure equation

$$
\frac{\beta p}{\rho}=1+\frac{2}{3} \pi \rho \int_{0}^{\infty} r^{3} g(r) \frac{\mathrm{d}[-\beta u(r)]}{\mathrm{d} r} \mathrm{~d} r .
$$

Imposing the equality between the repulsive contributions to the HCY and LJ potentials, on one hand, and between their attractive contributions, on the other hand, the mapping equations read explicitly

$$
\begin{array}{r}
\frac{2}{3} \pi \rho \sigma^{3} g_{\mathrm{HCY}}(\sigma, \lambda)=\frac{2}{3} \pi \rho \sigma_{\mathrm{LJ}}^{3} \int_{0}^{X_{m}} X^{3} g_{\mathrm{LJ}}(X) \frac{\mathrm{d}\left[-\beta u_{\mathrm{LJ}}(X)\right]}{\mathrm{d} X} \mathrm{~d} X, \\
\frac{2}{3} \pi \rho \int_{\sigma}^{\infty} r^{3} g_{\mathrm{HCY}}(r ; \sigma, \lambda) \frac{\mathrm{d}\left[-\beta u_{\mathrm{HCY}}(r)\right]}{\mathrm{d} r} \mathrm{~d} r=\frac{2}{3} \pi \rho \sigma_{\mathrm{LJ}}^{3} \int_{X_{m}}^{\infty} X^{3} g_{\mathrm{LJ}}(X) \frac{\mathrm{d}\left[-\beta u_{\mathrm{LJ}}(X)\right]}{\mathrm{d} X} \mathrm{~d} X,
\end{array}
$$

where the left-hand side of Eq. (23) corresponds to the rate of collision for the hard-sphere contribution of the HCY system. On the right side, $X\left(=r / \sigma_{\mathrm{LJ}}\right)$ is the reduced length and $X_{m}=2^{1 / 6}$ is the position of the minimum of the LJ potential. Then, the mapping procedure is performed by solving iteratively Eqs. (23) and (24) until both parameters $\sigma$ and $\lambda$ are obtained consistently. Practically, the right-hand sides of Eqs. (23) and (24) can be calculated as soon as $g_{\mathrm{LJ}}(r)$ is available as a function of $T$ and $\rho$.

\section{General features of the mapping parameters $\alpha$ and $\lambda$}

The purpose of the calculations reported here is to achieve an assessment of the mapping parameters $\alpha$ and $\lambda$ obtained by two different treatments. In using the first approach based on the simultaneous fitting of the virial pressure and excess internal energy of the HCY system into those of the LJ system, the inaccuracies of the numerical calculations have been greatly eliminated. Table 2 summarizes the behaviour of $\alpha$ and $\lambda$ over a wide range of state variables covering reduced densities $\rho^{*}$ from 0.1 up to 1 and temperatures from the triple point $\left(T^{*} \sim 0.8\right)$ 
KADIRI ET AL.: THERMODYNAMIC EQUIVALENCE BETWEEN THE LENNARD-JONES AND ...

up to $T^{*}=5$. Focusing our attention on $\alpha^{3}\left(=\sigma^{3} / \sigma_{\mathrm{LJ}}^{3}\right)$ to gain a feeling, we observe a weak variation of the effective hard-sphere diameter $\sigma$ compared to that of $\lambda$.

Table 2. Values of the parameters $\alpha^{3}$ and $\lambda$ for the HCY potential as functions of thermodynamic states, aimed to achieve the correspondence with the LJ fluid.

\begin{tabular}{|c|c|c|c|c|c|c|c|c|}
\hline$T^{*}$ & $\rho^{*} \rightarrow$ & 0.1 & 0.2 & 0.4 & 0.6 & 0.8 & 0.9 & 1 \\
\hline 5 & $\alpha^{3}$ & 0.72162 & 0.72920 & 0.74828 & 0.76540 & 0.77230 & 0.77410 & 0.77285 \\
& $\lambda$ & 1.5609 & 1.5968 & 1.7175 & 1.9520 & 2.4830 & 3.1900 & 5.0950 \\
\hline 3 & $\alpha^{3}$ & 0.80600 & 0.80425 & 0.81500 & 0.83230 & 0.84535 & 0.84632 & 0.84395 \\
& $\lambda$ & 1.5504 & 1.5603 & 1.6260 & 1.7475 & 2.0128 & 2.2745 & 2.7785 \\
\hline 2.5 & $\alpha^{3}$ & 0.83600 & 0.82920 & 0.84090 & 0.85825 & 0.87115 & 0.87235 & 0.86968 \\
& $\lambda$ & 1.5484 & 1.5553 & 1.6171 & 1.7215 & 1.9385 & 2.1457 & 2.5240 \\
\hline 2 & $\alpha^{3}$ & 0.88275 & 0.87600 & 0.87010 & 0.88601 & 0.90215 & 0.90490 & 0.90168 \\
& $\lambda$ & 1.5599 & 1.5729 & 1.6102 & 1.6985 & 1.8770 & 2.0462 & 2.3319 \\
\hline 1.6 & $\alpha^{3}$ & 0.95200 & 0.94000 & 0.91202 & 0.91745 & 0.93420 & 0.93755 & 0.93430 \\
& $\lambda$ & 1.6030 & 1.6050 & 1.6257 & 1.6942 & 1.8450 & 1.9855 & 2.2178 \\
\hline 1.4 & $\alpha^{3}$ & 1.02700 & 1.00720 & 0.94855 & 0.93810 & 0.95410 & 0.95702 & 0.95377 \\
& $\lambda$ & 1.6435 & 1.6445 & 1.6481 & 1.6997 & 1.8364 & 1.9610 & 2.1695 \\
\hline 1.2 & $\alpha^{3}$ & & & & 0.96120 & 0.97645 & 0.97955 & \\
& $\lambda$ & & & & 1.7075 & 1.8305 & 1.9430 & \\
\hline 1 & $\alpha^{3}$ & & & & & 1.00175 & 1.00686 & \\
& $\lambda$ & & & & & 1.8287 & 1.9331 & \\
\hline 0.8 & $\alpha^{3}$ & & & & & 1.03495 & 1.03990 & \\
& $\lambda$ & & & & & & & \\
\hline
\end{tabular}

This emphasizes the role of the repulsive forces in establishing the thermodynamic properties of liquids, since the particles are so close to each other that a small increase of their size produces a large increment of the pressure. The parameter $\alpha$, almost always smaller than one, depends on both $T^{*}$ and $\rho^{*}$. While it is clearly inversely dependent on temperature, its density dependence seems to be more complicated with no detectable general trend. The parameter $\lambda$, characteristic of the attractive region of the HCY potential, depends strongly on $T^{*}$ and $\rho^{*}$. It always increases with density, whatever the temperature, but its variation as a function of temperature is different at low and high densities: $\lambda$ decreases with increasing $T^{*}$ at low densities, whereas the situation is reversed at high densities. This behaviour is consistent with the observation of Ashcroft [25] assessing that the HCY system passes from a supercritical fluid phase to a solid phase as the density increases. It is worth knowing that, at $T^{*}=5$, the parameter $\lambda$ is about 15 for $\rho^{*}=1.1$ and 
becomes infinite for $\rho^{*}=1.2$ with the impossibility to reach the exact value of the excess internal energy $(+0.370)$. Note that Hagen and Frenkel [3] have obtained the disappearance of the stable liquid phase for the significantly lower value of $\lambda=7.4$ using a Monte-Carlo based perturbation theory. The values of $\alpha^{3}\left(\rho^{*}, T^{*}\right)$ and $\lambda\left(\rho^{*}, T^{*}\right)$ displayed in Table 2 can be adequately represented as a function of temperature by the following parametric expressions

$$
\begin{aligned}
\alpha^{3}\left(T^{*}\right) & =A_{0}\left(\rho^{*}\right)+A_{1}\left(\rho^{*}\right) T^{*}+A_{2}\left(\rho^{*}\right) T^{* 2}+A_{3}\left(\rho^{*}\right) T^{* 3} \\
\lambda\left(T^{*}\right) & =B_{0}\left(\rho^{*}\right)+B_{1}\left(\rho^{*}\right) T^{*}+B_{2}\left(\rho^{*}\right) T^{* 2}+B_{3}\left(\rho^{*}\right) T^{* 3},
\end{aligned}
$$

where the parameters $A_{i}\left(\rho^{*}\right)$ and $B_{i}\left(\rho^{*}\right)$ obtained by least squares-fitting are given in Table 3.

Table 3. Values of the coefficients $A_{i}\left(\rho^{*}\right)$ and $B_{i}\left(\rho^{*}\right)$ of the polynomial expressions given by Eqs. (25) and (26).

\begin{tabular}{|c|c|c|c|c|c|c|c|c|}
\hline$\rho^{*}$ & $A_{0}$ & $A_{1}$ & $A_{2}$ & $A_{3}$ & $B_{0}$ & $B_{1}$ & $B_{2}$ & $B_{3}$ \\
\hline 0.2 & 1.7345 & -0.8057 & 0.2321 & -0.0226 & 2.1276 & -0.5540 & 0.1700 & -0.0161 \\
0.4 & 1.3255 & -0.4037 & 0.1088 & -0.0102 & 1.9286 & -0.3333 & 0.1071 & -0.0098 \\
0.6 & 1.1529 & -0.2127 & 0.0478 & -0.0042 & 1.8255 & -0.1638 & 0.0587 & -0.0042 \\
0.8 & 1.1848 & -0.2127 & 0.0478 & -0.0042 & 1.9091 & -0.1457 & 0.0729 & -0.0042 \\
1 & 1.1924 & -0.2335 & 0.0540 & -0.0048 & 1.7734 & 0.3878 & -0.1265 & 0.0364 \\
\hline
\end{tabular}

In the second approach, the parameters $\alpha$ and $\lambda$ can be determined using the concept of collision frequency. Equations (23) and (24) allow to separate the contributions of repulsive and attractive forces to the virial pressure. For the purpose, the repulsive and attractive contributions to the virial pressure of the LJ potential have been calculated with the pair-correlation function obtained in molecular dynamics (MD). The MD simulations were performed in the NVT ensemble with $N=256$ particles. The system was set in a fcc lattice in cubic boxes and surface effects were avoided by placing each box at the centre of a periodic array of identical boxes. The LJ potential was truncated at intermolecular separations greater than $r_{c}=2.5 r_{m}$, corresponding to half the box length. Appropriate long-range corrections were used to recover the full contribution to the intermolecular potential [26]. The equations of motion were integrated using Verlet's algorithm in the velocity form with a time step of $\Delta t=10^{-15} \mathrm{~s}$ and the pair-correlation function $g(r)$ was extracted from a sample of $4 \times 10^{4}$ configurations every $10 \Delta t$ after an equilibration of $10^{4}$ steps. In contrast, the pair-correlation function of the HCY system, necessary to the calculation of the virial pressure, has been determined with the analytical expression derived by Henderson et al. [10] as it has been shown to provide very accurate $g_{\mathrm{HCY}}(r ; \sigma, \lambda)$ and because it is less time-consuming than the MD simulation.

In this mapping procedure, everything amounts to finding $\alpha$ and $\lambda$ by solving iteratively Eqs. (23) and (24) for each temperature and density. It is found that 
KADIRI ET AL.: THERMODYNAMIC EQUIVALENCE BETWEEN THE LENNARD-JONES AND ...

the numerical effort involved in this procedure is not as great as expected because Eq. (23) is rather insensitive to the parameter $\lambda$, whereas Eq. (24) is insensitive to the parameter $\alpha$. This treatment has been only performed for three temperatures, $T^{*}=1.4,1.6$ and 2 in order to compare the two procedures of mapping. The parameters corresponding to the collision frequency treatment, labelled $\alpha_{2}$ and $\lambda_{2}$, are collected in Table 4 together with those coming from the simultaneous fitting of the virial pressure and excess internal energy, labelled $\alpha_{1}$ and $\lambda_{1}$. The general trends of both sets of results are qualitatively the same. For the parameter $\alpha$, the agreement is excellent at the lowest temperature $\left(T^{*}=1.4\right)$ and still reasonable at $T^{*}=2$ with a difference of about $8 \%$ for $\rho^{*}=0.2$. Concerning the parameter $\lambda$, the concordance is particularly good for the highest temperature whatever the density, but deteriorates at high density and low temperature when the critical temperature is approached. On the whole, there is a reasonable homogeneity in the results of the parameters $\alpha$ and $\lambda$ for both procedures of mapping.

Table 4. Values of the parameters $\alpha^{3}$ and $\lambda$ for the HCY potential as functions of thermodynamic states, derived to achieve the correspondence with the LJ fluid. The index 1 stands for the direct mapping procedure and the index 2 is for the mapping that involves the collision frequency concept. The contributions of repulsive and attractive forces to the virial pressure are also displayed.

\begin{tabular}{|c|c|c|c|c|c|c|c|}
\hline$T^{*}$ & $\rho^{*}$ & $\frac{\beta p_{\mathrm{LJ}}}{\rho}(\mathrm{rep})$ & $\frac{\beta p_{\mathrm{LJ}}}{\rho}(\mathrm{att})$ & $\alpha_{2}^{3}$ & $\lambda_{2}$ & $\alpha_{1}^{3}$ & $\lambda_{1}$ \\
\hline 1.4 & 0.2 & 1.87169 & -1.29949 & 1.010 & 1.646 & 1.00720 & 1.6445 \\
& 0.4 & 2.82476 & -2.43903 & 0.947 & 1.648 & 0.94855 & 1.6481 \\
& 0.6 & 4.23981 & -3.50765 & 0.922 & 1.718 & 0.93810 & 1.6997 \\
& 0.8 & 7.00525 & -4.33991 & 0.930 & 1.978 & 0.95410 & 1.8364 \\
& 0.9 & 9.33909 & -4.59601 & 0.934 & 2.268 & 0.95702 & 1.9610 \\
& 1 & 12.72284 & -4.84894 & 0.936 & 2.780 & 0.95377 & 2.1695 \\
\hline 1.6 & 0.2 & 1.80186 & -1.13651 & 0.985 & 1.622 & 0.94000 & 1.6050 \\
& 0.4 & 2.70906 & -2.09816 & 0.927 & 1.625 & 0.91202 & 1.6257 \\
& 0.6 & 4.14577 & -3.12830 & 0.919 & 1.692 & 0.91745 & 1.6942 \\
& 0.8 & 6.78998 & -3.8283 & 0.921 & 1.935 & 0.93420 & 1.8450 \\
& 1 & 12.29784 & -4.46279 & 0.929 & 2.386 & 0.93430 & 2.2178 \\
\hline 2 & 0.2 & 1.71470 & -0.89083 & 0.955 & 1.587 & 0.87600 & 1.5729 \\
& 0.4 & 2.57347 & -1.68103 & 0.907 & 1.594 & 0.87010 & 1.6102 \\
& 0.6 & 3.92339 & -2.44415 & 0.896 & 1.676 & 0.88601 & 1.6985 \\
& 0.8 & 6.42608 & -3.08320 & 0.902 & 1.878 & 0.90215 & 1.8770 \\
& 1 & 11.13069 & -3.44213 & 0.901 & 2.358 & 0.90168 & 2.3319 \\
\hline
\end{tabular}

FIZIKA A (Zagreb) 16 (2007) 2, 47-62 
The evaluation of not only the EOS but also of the corresponding thermodynamic properties is the ultimate test to judge the accuracy of the parameters $\alpha$ and $\lambda$. Starting from the expressions for $\beta A / N, \beta U^{e x} / N$ and $\beta p / \rho$ given, respectively, by Eqs. (3), (14) and (15), the excess thermodynamic quantities (excess free energy, excess entropy and excess chemical potential) related to them are commonly evaluated using the relationships

$$
\begin{aligned}
\frac{\beta A^{e x}}{N} & =\frac{\beta A}{N}-\left[\ln \Lambda^{3} \rho-1\right], \\
\frac{S^{e x}}{N k_{B}} & =\frac{\beta U^{e x}}{N}-\frac{\beta A^{e x}}{N}, \\
\beta \mu^{e x} & =\frac{\beta A}{N}+\frac{\beta p}{\rho}-\ln \Lambda^{3} \rho .
\end{aligned}
$$

Because in the first mapping procedure the excess internal energy and virial pressure were forced to fit simultaneously the reference values, all excess quantities coincide perfectly with the MD data. On the contrary, in the second mapping procedure, only the pressure fits the MD data. Therefore, we show in Fig. 2 the excess
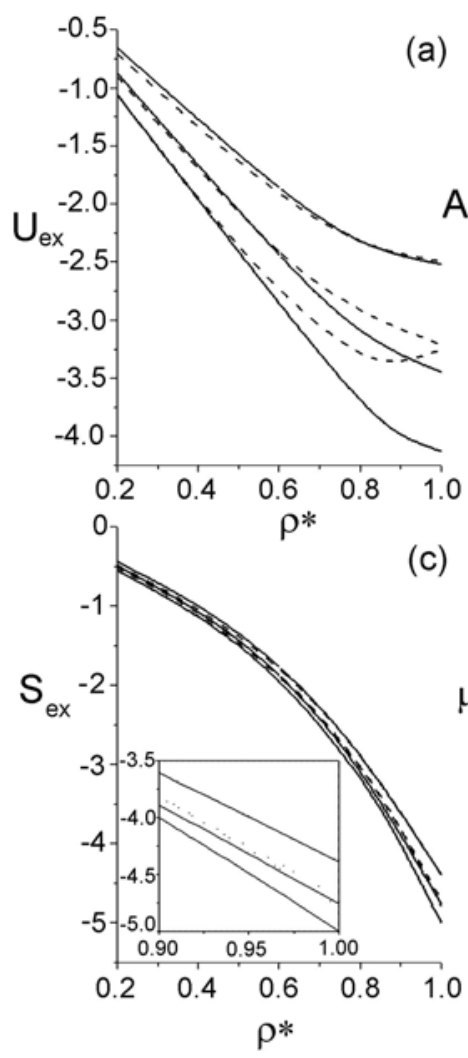

58
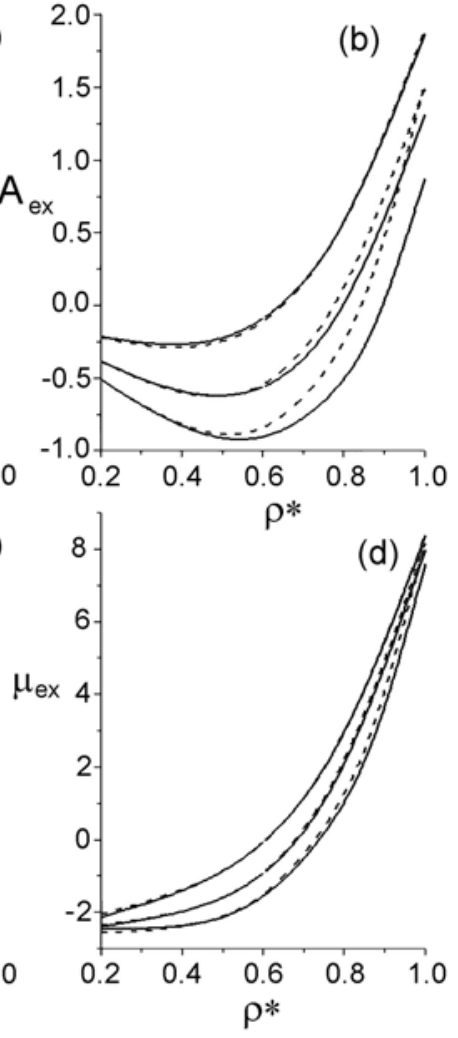

Fig. 2. Thermodynamic quantities of the $\mathrm{HCY}$ system for the three isotherms $T^{*}=1.4,1.6$ and 2 (from bottom to top). Full line corresponds to the reference data of the LJ fluid, dotted line is for the results derived by the mapping procedure that involves the collision frequency concept. 
quantities along the isotherms $T^{*}=1.4,1.6$ and 2 in order to judge the quality of the mapping procedure based on the collision frequency. On the whole, the excess internal energy $\beta U^{e x} / N$ (Fig. 2a), the excess free energy $\beta A^{e x} / N$ (Fig. 2b), the excess entropy $S^{e x} / N k_{B}$ (Fig. 2c) and the excess chemical potential $\beta \mu^{e x}$ (Fig. $2 \mathrm{~d})$ are in quite good agreement with the reference data. As expected, the worst result is observed especially for the excess internal energy and excess free energy for $T^{*}=1.4$ and $\rho^{*}=1$, where the values of the parameters $\lambda_{2}$ and $\lambda_{1}$ are slightly different. In contrast, the excess entropy is hardly affected since it is the difference between two quantities of equal magnitude $\left(\beta U^{e x} / N-\beta A^{e x} / N\right)$.

\section{Concluding remarks}

The investigation of the thermodynamic properties of the LJ fluid has been made for a system of particles interacting with the HCY potential. The method is advantageous due to a convenient analytical expression for the equation of state for the HCY system derived in the mean spherical approximation. Specifically, the method consists in mapping the thermodynamic properties of the LJ fluid, for every $\rho^{*}$ and $T^{*}$, from the EOS of the equivalent HCY system for suitable values of its two parameters $\alpha$ and $\lambda$. An advantage of this approach is that it allows to predict the thermodynamic properties of the LJ fluid by means of only two molecular parameters.

In order to establish the particularities of the dependence of $\alpha$ and $\lambda$ on $\rho^{*}$ and $T^{*}$, two mapping procedures have been employed. The first one requires the equality of the virial pressure and internal energy of the LJ fluid with those of the HCY system, and in the second one the repulsive and attractive contributions to the virial pressure are kept the same in the LJ and HCY systems. In both procedures, the equations are solved iteratively and provide unique solutions in any thermodynamic state.

To sum up the work, we point out that the results are threefold: (i) the EOS of the HCY system can be used to predict the thermodynamic properties of the LJ fluid over a wide domain of the phase diagram, (ii) the concept of collision frequency can be used confidently to map any system into another one provided that both pair-correlation functions are available and (iii) the EOS of the HCY system is capable to connect rigorously its own parameters to the properties of a real fluid, without reference to the LJ potential. Therefore, the HCY system is a good reference system.

Firstly, comparison of our predictions of the thermodynamic properties of the LJ fluid with reference data show that the analytical EOS of the HCY system is highly reliable when the correct values of the parameters are used. In particular, this analytical EOS is of precision comparable to those of the integral equation [27] or simulation data for the LJ fluid. Compared to the strictly empirical equations of state using a modified form of the Benedict-Webb-Rubin equation (MBWR) [28], the EOS of the HCY system has only two state dependent parameters, $\alpha$ and $\lambda$, instead of 33 parameters of the MBWR equation of state. Taking account of

FIZIKA A (Zagreb) 16 (2007) 2, 47-62 
the physical meaning of $\alpha$ and $\lambda$, their extrapolation allows to predict additional thermodynamic properties outside the range for which the parameters have been determined.

Secondly, the reasonable homogeneity in the values of the parameters $\alpha$ and $\lambda$ for both procedures of mapping promotes the treatment based on the collision frequency concept, even if the direct fitting of the virial pressure and internal energy is expected to provide the best EOS. The parameter values obtained with the procedure that involves the collision frequencies somewhat differ from those evaluated within the simultaneous fitting of the virial pressure and internal energy, but the general shapes of their density and temperature dependence remain very similar to each other. In particular, the variations of $\alpha$ and $\lambda$ with temperature are easily parametrized smooth functions, while the density dependence of $\alpha$ is much more structured with an evident non-monotonicity in the relevant region of density. Besides, the former mapping procedure involves explicitly the HCY pair-correlation function, $g_{\mathrm{HCY}}(r)$, and the latter ignores it by setting up an analytical free-energy expression for the HCY system. The confidence in the behaviour of $g_{\mathrm{HCY}}(r)$ is then replaced by implicitly estimated energetic and entropic effects. As a result, the precise shape of the pair-correlation function is not so important for the prediction of the thermodynamics of the LJ system in the low-density region.

Thirdly, the main issue of the work is the proof that the HCY system is a very good reference system, owing to the quite accurate expressions for the free energy and the pair-correlation function, which offers the possibility to implement the variational method in order to determine the thermodynamic properties of fluids with realistic potentials. Alternatively, it can be used more directly to approximate the best available rare gas potential without going through the LJ potential as an intermediate approximation. It should also be mentioned that the inverse screening length $\lambda$ increases beyond the commonly accepted value of 1.8 for the LJ fluid as the density and temperature are increasing. Such high values of $\lambda$ correspond to very short-range potentials, a situation in which the phase diagram of the fluid undergoes profound modifications with no stable liquid phase among them [3]. Even if the present treatment is still not flexible enough to describe fluids with nonspherical potentials and mixtures, it is believed to represent simple and useful means of solving the van der Waals problem of an accurate analytical EOS based on underlying theory.

\section{Acknowledgements}

The authors are very grateful to Carlos Rey for providing the MD code for the HCY potential.

References

[1] D. A. McQuarrie, Statistical Mechanics, Harper \& Row Publishers, New York (1976).

[2] J. P. Hansen and I. R. McDonald, Theory of Simple Liquids, Academic Press, London (1986). 
KADIRI ET AL.: THERMODYNAMIC EQUIVALENCE BETWEEN THE LENNARD-JONES AND ...

[3] M. H. J. Hagen and D. Frenkel, J. Chem. Phys. 101 (1994) 4093.

[4] E. Waisman, Mol. Phys. 25 (1973) 45.

[5] J. S. Hoye and G. Stell, Mol. Phys. 32 (1976) 195; 32 (1976) 209.

[6] P. T. Cummings and E. R. Smith, Mol. Phys. 38 (1979) 997.

[7] M. Ginoza, J. Phys. Soc. Jpn. 54 (1985) 2783; Mol. Phys. 71 (1990) 145.

[8] D. Henderson, G. Stell and E. Waisman, J. Chem. Phys. 62 (1975) 4247.

[9] D. Henderson, L. Blum and J. P. Noworyta, J. Chem. Phys. 102 (1995) 4973.

[10] D. Henderson, L. Mier-Y-Teran and L. Blum, Fluid Phase Equilibria 130 (1997) 65.

[11] F. del Rio, Mol. Phys. 42 (1981) 217.

[12] G. A. Mansoori, Fluid Phase Equilibria 13 (1983) 153.

[13] K. Aim and I. Nezbeda, Fluid Phase Equilibria 12 (1983) 235; 17 (1984) 1.

[14] Y. Song and E. A. Mason, J. Chem. Phys. 91 (1989) 7840.

[15] L. Verlet and J. J. Weis, Phys. Rev. A 5 (1972) 939.

[16] A. Gil-Villegas and F. del Rio, Phys. Rev. E 53 (1996) 2326.

[17] Y. V. Kalyuzhnyi and P. T. Cummings, Mol. Phys. 87 (1996) 1459.

[18] T. Boublik, Mol. Phys. 59 (1986) 371.

[19] N. F. Carnahan and K. E. Starling, J. Chem. Phys. 51 (1969) 635.

[20] J. J. Erpenbeck and W. W. Wood, J. Stat. Phys. 35 (1984) 321.

[21] D. M. Duh and L. Mier-Y-Teran, Mol. Phys. 90 (1997) 373.

[22] D. Henderson, E. Waisman, J. L. Lebowitz and L. Blum, Mol. Phys. 35 (1978) 241.

[23] K. S. Pitzer, J. Chem. Phys. 7 (1939) 583; J. Am. Chem. Soc. 77 (1955) 3427.

[24] F. del Rio, Mol. Phys. 76 (1991) 21; F. del Rio and A. Gil-Villegas, Mol. Phys. 77 (1992) 223.

[25] N. W. Ashcroft, Nature 365 (1993) 387.

[26] Y. Kadiri, Thèse de l'Université de Metz, 2001.

[27] J. M. Bomont and J. L. Bretonnet, J. Chem. Phys. 114 (2001) 4141; Mol. Phys. 101 (2003) 3249.

[28] J. K. Johnson, J. A. Zollweg and K. E. Gubbins, Mol. Phys. 78 (1993) 591. 


\section{TERMODINAMIČKA JEDNAKOVALJANOST LENNARD-JONESOVOG SUSTAVA I PRIVLAČNOG YUKAWINOG SUSTAVA S TVRDOM SREDICOM}

Istražujemo termodinamička svojstva Lennard-Jonesove (LJ) tekućine proučavanjem sustava čestica koje međudjeluju potencijalom tvrda sredica i privlačan Yukawin rep (HCY). Zbog sličnosti LJ i HCY oblika potencijala, vrijedi tražiti približenje LJ potencijalu kao što se rabi referentni potencijal tvrdih kuglica. U ovom proučavanju opisujemo termodinamiku LJ tekućine pomoću jednakovaljanog HCY sustava čija su svojstva točno poznata računanjem termodinamičkih veličina za parametre HCY potencijala. Ta je metoda izvediva zbog pogodnog izraza za Helmholtzovu slobodnu energiju u prosječno-sfernom približenju, razvijenu po potencijama recipročne temperature. Rabe se dva postupka za određivanje parametara HCY potencijala kao funkcije termodinamičkog stanja: jedan se zasniva na istovremenoj prilagodbi tlaka i unutarnje energije LJ sustava, a drugi rabi zamisao sudarne frekvencije. Dobra jednolikost ishoda oba postupka snimanja čini HCY potencijal vrlo dobrim referentnim sustavom čiji se teorijski izrazi mogu s pouzdanošću rabiti za predviđanje termodinamičkih svojstava sustava sa stvarnim potencijalima. 